Kardiologe $2021 \cdot 15: 272-281$

https://doi.org/10.1007/s12181-021-00482-9

Angenommen: 3. März 2021

Online publiziert: 18. Mai 2021

(c) Deutsche Gesellschaft für Kardiologie -

Herz- und Kreislaufforschung e.V. Published by

Springer Medizin Verlag $\mathrm{GmbH}$, ein Teil von

Springer Nature - all rights reserved 2021

\author{
Christian Butter ${ }^{1}$ Johannes Sperzel ${ }^{2}$ \\ 'Immanuel Klinikum Bernau Herzzentrum Brandenburg, Universitätsklinikum der Medizinischen \\ Hochschule Brandenburg, Bernau bei Berlin, Deutschland \\ ${ }^{2}$ Abteilung für Kardiologie, Kerckhoff-Klinik GmbH, Bad Nauheim, Deutschland
}

\title{
Gerätebasierte rhythmologische Diagnostik und Therapie in COVID-19-Zeiten
}

dem Drang der Publikation schritthalten konnte [1].

Über die Umverteilung der finanziellen Ressourcen hinaus haben auch die Reisebeschränkungen, die reduzierte Betreuung von Studien durch Spezialisten und die Einschränkungen in den Behandlungsmöglichkeiten von sog. „nicht akuten“ Patienten Auswirkungen auf die Durchführung von kardiologischen Studien. Patienten haben sich aus Sorge vor Ansteckung mit dem Coronavirus von Kliniken ferngehalten, haben sich spät im Krankheitsverlauf vorgestellt und haben ambulante Nachbeobachtungstermine sowohl in Kliniken und Praxen vermieden, sodass u. a. auch bereits laufende kardiologische Studien weniger Einschlüsse zu verzeichnen hatten und haben.

\section{Rhythmologische Betreuung in Pandemiezeiten}

Mit Beginn der Pandemie zu Beginn des letzten Jahres ist frühzeitig die Diskussion um die Dringlichkeit und Notwendigkeit medizinischer Eingriffe in der Nutzen-Risiko-Abwägung in der allgegenwärtigen Infektsituation entbrannt. Die Umverteilung personeller Ressourcen innerhalb der Kliniken zugunsten der Behandlung von COVID-Erkrankten, die Bereitstellung von räumlichen Kapazitäten, von Beatmungsmöglichkeiten und Intensivbetten hat ein Umdenken in der etablierten Einbestellungs- und Behandlungspraxis von kardiologischen Patienten nach sich gezogen. Eine Graduierung nach Dringlichkeit und Notwendigkeit medizinischer Eingriffe wurde eingefordert mit der Konsequenz, bestimmte Patienten nicht zu versorgen, was gerade in der Herz-Kreislauf-Medizin mit nicht abschätzbaren Folgen einer unterlassenen Behandlung als sehr kritisch einzuschätzen ist. Im September 2020 haben die Heart Rhythm Society COVID-19 Task Force, die Electrophysiology Section des American College of Cardiology und das Electrocardiography and Arrhythmias Committee des Council on Clinical Cardiology der American Heart Association eine Richtlinie veröffentlicht, die eine Hilfestellung bei der Graduierung der Dringlichkeit von rhythmologischen Interventionen und Eingriffen inklusive der Device-Therapie und Nachsorge gibt, die vorrangig dem Schutz von $\mathrm{Pa}$ tienten, Angehörigen des Gesundheitswesens, Industriemitarbeitern und Krankenhausangestellten dienen soll [2].

In dieser Richtlinie wird zwischen „urgent/emergent", „semi-urgent“ und "non-urgent/elective“ unterschieden. Behandelt werden sollten somit in jedem Fall die dringlich einzuschätzenden Indikationen, unterlassen werden sollten Eingriffe/Interventionen mit nichtdringlicher/elektiver Notwendigkeit.

Im Bereich der elektrischen Implantate werden folgende Eingriffe als dringend notwendig eingestuft:

- Sondenrevisionen bei stimulationsabhängigen Schrittmacherpatienten und/oder inadäquate Defibrillatortherapie bei ICD(implantierbarer Kardioverter-Defibrillator)-Trägern, 
- Batteriewechsel bei elektiver Austauschindikation oder bei Batterieerschöpfung („end of life“),

- Batterieerschöpfung mit minimaler Restlaufzeit unter besonderen Situationen,

- ICD-Implantation zur Sekundärprävention,

- Schrittmachernotwendigkeit bei AV(atrioventrikulär)-Block III; Mobitz II oder höhergradiger AV-Block mit Symptomen sowie symptomatische Sinusknotendysfunktion mit langen Pausen,

- antibiotikaresistente Sonden-/ Device-Infektion oder Endokarditis, Bakteriämie oder Tascheninfektion,

- CRT(kardiale Resynchronisationstherapie)-Indikation bei therapieresistenter schwerer Herzinsuffizienz unter optimaler leitlinienkonformer medikamentöser Therapie.

Demgegenüber wird ein sehr zurückhaltendes und vermeidendes Verhalten bei den nichtdringlichen oder elektiven Indikationen empfohlen, wozu folgende Maßnahmen zählen:

- ICD-Implantation zur Primärprävention,

- CRT bei stabilen Patienten,

- Upgrades verschiedener Systeme,

- Schrittmacherimplantationen bei Sinusknotendysfunktion, AV-Block Mobitz I, wenig symptomatische Brady-Tachy-Syndrome,

- ICD-Batteriewechsel bei einer Restlaufzeit von mehr als 6 Wochen,

- Entfernung/Extraktion nicht infizierter Sonden/Aggregate, es sei denn, die Device-Funktion ist beeinträchtigt.

$\mathrm{Zu}$ den zu vermeidenden Interventionen gehören u.a. auch die Diagnostik supraventrikulärer Tachykardien und die Ablation atrialer Arrhythmien inklusive Vorhofflimmerablationen, solange die Patienten stabil sind. Grundsätzlich gibt es einen klinischen Ermessensspielraum, der sich auch in der Kategorie „semiurgent" niederschlägt. Das Patientenund das Mitarbeiterwohl sollten immer im Licht einer angemessenen Versorgung und notwendiger Vermeidung/ Ausbreitung von Infektionen beurteilt werden.

\section{Device-Nachsorge}

Auch in der Device-Nachsorge sollen so wenig wie möglich Patienten die Kliniken oder Praxen betreten und die persönlichen Kontakte vermieden werden, wenn machbar. Grundsätzlich und theoretisch wäre zumindest ein Teil der Implantatträger, die mit modernen telemedizinfähigen Geräten versorgt sind, per „remote interrogation“ $\mathrm{zu}$ verfolgen und ein Teil der Routinenachsorge zu vermeiden. Ebenso könnte eine Bildübermittlung von z.B. einer suspekten Schrittmacher-/Defibrillatortasche aus der Häuslichkeit erfolgen. Über welche Plattform das dann zu geschehen hätte und wie gerade ältere Schrittmacherpatienten das technisch bewältigen könnten, bleibt auch unter datenschutzkonformem Vorgehen zurzeit ungeklärt.

Übrig bleiben und empfohlen werden somit nur Nachsorgekontakte aus folgenden dringlichen oder zeitkritischen Indikationen:

- Auffälligkeiten der Devices im Remote-Monitoring, in der Telemetrie oder im ambulanten Monitoring, die eine klinische Gefährdung oder Konsequenz nach sich ziehen,

- ICD-Schocks, Synkope oder Präsynkope in Zusammenhang mit einem arrhythmischen Ereignis, die eine Umprogrammierung erfordern,

- verdächtige Symptome oder Auffälligkeiten im Remote-Monitoring, die in Zusammenhang mit einer Fehlfunktion oder Fehlprogrammierung stehen könnten,

- Notwendigkeit einer Umprogrammierung,

- verdächtige Symptome bei DeviceTrägern, die keine Remote-Monitoring-Funktion haben,

- Notwendigkeit einer dringlichen nichtvermeidbaren MRT (Magnetresonanztomographie)-Untersuchung.

Obwohl das Remote-Monitoring schon seit 2015 eine Klasse-I-Indikation aufweist [3], wird gerade in den aktuellen Pandemiezeiten deutlich, dass es nach wie vor an einer strukturierten flächendeckenden Umsetzung mangelt. Die Ursachen sind vielfältig und seit Jahren bekannt. Hierzu gehören eine uneinheitli- che technische Ausstattung, firmenspezifische Unverträglichkeiten und Besonderheiten, fehlende Plattformen, unklare Organisationsabläufe und Zuständigkeiten sowie ein fragwürdiges und noch $\mathrm{zu}$ klärendes Entgeltsystem, das bisher über Jahre nicht refinanzierte etablierte telemedizinische Strukturen in Kliniken in den bestehenden Organisationsformen nicht unterstützt hat.

\section{Auswirkungen auf die klinische Versorgung - am Beispiel Italiens}

Bereits vor Erscheinen dieser Empfehlungen hat in Italien, das in der ersten Phase der Pandemie besonders heftig betroffen war, eine Priorisierung von rhythmologischen Interventionen und Eingriffen stattgefunden. Eine so differenzierte Einteilung - wie oben ausgeführt - lag jedoch für die dortige kardiologische Versorgung noch nicht vor, wie eine Umfrage der Italian Association of Arrhythmology and Cardiac Pacing zeigte [4]. So schien die Versorgung von Patienten mit Rhythmusimplantaten während des Lockdowns gravierend eingeschränkt $\mathrm{zu}$ sein [5]. Basierend auf dieser Befragung von 104 Ärzten aus 84 rhythmologischen Zentren, die damit fast ein Viertel der vorhandenen Rhythmologien darstellen, haben sich im Zeitraum von Ende April bis Ende Mai 2020 im Vergleich zum identischen Vorjahreszeitraum bedeutsame Veränderungen ergeben. Die Mehrzahl der Befragten berichtet einen einschneidenden Rückgang der elektiven Schrittmacherimplantationen, 50\% von ihnen sogar einen Rückgang von über $50 \%$. Eine ähnliche Abnahme zeigt sich auch bei den ICD-Implantationen zur Primärprävention; $50 \%$ der Befragten gaben sogar eine Abnahme ihrer Implantationszahlen von mehr als $65 \%$ an. Der Rückgang bei der Sekundärprävention scheint geringer zu sein. Etwa $25 \%$ bemerkten keine Veränderung bei dringlicher Indikation. Die Auswirkungen auf das Nachsorgeverhalten sind uneinheitlicher. Während fast die Hälfte eine unveränderte Nutzung per RemoteMonitoring berichtet, gibt ein Drittel 
Hier steht eine Anzeige.

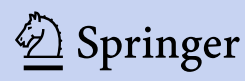


Kardiologe 2021 - 15:272-281 https://doi.org/10.1007/s12181-021-00482-9

(c) Deutsche Gesellschaft für Kardiologie - Herz- und Kreislaufforschung e.V. Published by Springer Medizin Verlag GmbH, ein Teil von Springer Nature all rights reserved 2021

\section{Butter ·. Sperzel}

\section{Gerätebasierte rhythmologische Diagnostik und Therapie in COVID-19-Zeiten}

\section{Zusammenfassung}

Die COVID-Pandemie mit ihrer ersten und zweiten Welle hat uns gezwungen, über eine sinnvolle Nutzung unserer medizinischen Ressourcen nachzudenken und Eingriffe nach ihrer Notwendigkeit und Dringlichkeit zu graduieren. Diese Selektion ist insbesondere in der Kardiologie in jedem einzelnen Fall schwierig und riskant. Die aktuellen Empfehlungen in der Herzschrittmacher-, Defibrillatortherapie und Nachsorge werden aufgezeigt mit dem Ziel, die individuelle ärztliche Entscheidung auf nachvollziehbare und belastbare Argumente zu stützen und diese auch den Patienten gegenüber begründen zu können. Diese Überlegungen haben in einzelnen Ländern zu einem deutlichen Rückgang von Schrittmacherund Defibrillatorimplantationen geführt ebenso wie Ablation bei supraventrikulären Arrhythmien. Die Auswirkungen der Pandemie lassen sich aus den Aktivitätsprofilen der rhythmologischen Implantate nachvollziehen. Die Notwendigkeit und Chancen einer telemedizinischen Überwachung sind in dieser Zeit nachdrücklich deutlich geworden, ebenso wie die bislang unzureichende Nutzung und mangelnde Struktur. Die aktuelle positive G-BA(Gemeinsamer Bundesausschuss)-Bewertung der telemedizinischen Überwachung bei Herzinsuffizienz weist in die richtige Richtung, die Umsetzung und Vergütung wird jedoch für weitere Diskussionen sorgen. Ein neuer Algorithmus, der auf einer Herztonerkennung beruht und im ersten AV(atrioventrikulären)sequenziellen sondenlosen Herzschrittmacher Verwendung findet, wird diskutiert. Bisher stützen sich die Indikationen für eine primärprophylaktische ICD(implantierbarer Kardioverter-Defibrillator)-Implantation im Wesentlichen auf die linksventrikuläre Ejektionsfraktion insbesondere bei der nichtischämischen Kardiomyopathie. Die Notwendigkeit und Bedeutung von intramuralem Fibrose- und Narbennachweis in der Magnetresonanztomographie (MRT) werden diskutiert und könnten die Entscheidungsfindung unterstützen.

Schlüsselwörter Patientenselektion · Sondenloser Herzschrittmacher · Telemedizin · Fibrose bei plötzlichem Herztod · Defibrillatortherapie

\section{Device-based rhythmological diagnostics and treatment in times of COVID-19}

\section{Abstract}

The first and second waves of the coronavirus disease 2019 (COVID-19) pandemic has deeply influenced our thinking on medical resources and forced us to grade interventions according to necessity and priority. In cardiology this selection is difficult and risky in every individual case. The current recommendations on pacemaker and defibrillator treatment as well as aftercare are presented with the aim of supporting the individual medical decisions with comprehensible and reliable arguments and to be able to justify these to the patients. In individual countries these considerations have led to a clear reduction in the implantation of pacemakers and defibrillators and also of ablation in cases of supraventricular arrhythmia. The impacts of the pandemic can be comprehended from the activity profiles of rhythmological implants. The necessity and opportunities for telemedical surveillance have become abundantly clear during this period as has the insufficient use and lack of structure of this technology. The current positive assessment by the Federal Joint Committee (G-BA) for telemedical monitoring in cardiac insufficiency is a step in the right direction; however, the implementation and remuneration will lead to further discussions. A new algorithm, which is based on the recognition of heart sounds and is used in the first atrioventricular (AV) leadless cardiac pacemaker, is discussed. So far, the indications for a primarily prophylactic implantation of an implantable cardioverter defibrillator (ICD) have essentially been based on the left ventricular ejection fraction, especially in nonischemic cardiomyopathy. The necessity and importance of detection of intramural fibrosis and cicatrices in magnetic resonance imaging (MRI) are discussed and could support decision-making.

\section{Keywords}

Patient selection - Leadless pacemaker . Remote monitoring · Scar in sudden cardiac death . Defibrillator treatment an, die Remote-Monitoring-Funktion vermehrt genutzt zu haben.

Unklar und bislang nicht beschrieben sind die Auswirkungen der zweiten Welle der Pandemie. Die beschriebenen notwendigen Veränderungen im Umgang mit der Nachbetreuung sowie der grundsätzlichen Nutzen von RemoteMonitoring-fähigen Geräten heben den besonderen Wert dieser Technologie hervor und machen deutlich, dass wir zeitnah und mit Nachdruck die Grundlagen finanzieller, technischer und struktureller Art legen müssen.

\section{Informationen aus Implantaten im Lockdown}

In einer kleinen italienischen Studie wurden an 24 Patienten die Auswirkungen des 40-tägigen strikten Lockdowns auf die körperliche Aktivität untersucht [5]. Via Home-Monitoring wurden bei ICDTrägern, die aus primärprophylaktischer Indikation versorgt worden sind, die Ak- zelerometerdaten mit dem vergleichbaren Zeitintervall vor dem Lockdown verglichen. Bedingt durch geschlossene Fitnessstudios, fehlende Physiotherapie, Beschränkung auf die Häuslichkeit und eine strikte Ausgangssperre zeigte sich eine Abnahme der körperlichen Aktivität um $25 \%$, was bei den ohnehin eingeschränkt belastbaren und beweglichen Patienten weitere ungünstige Auswirkungen haben dürfte und ermutigen sollte, auch „indoor" ein strukturiertes Trainingsprogramm aufrechtzuerhalten [5]. 
Device-basierte Telemedizin kann helfen, neben einer Progredienz der Herzinsuffizienz auch Änderungen der Lebensumstände $\mathrm{zu}$ erfassen und entsprechend frühzeitig zu reagieren. Diese Informationen werden unterstützt durch die Analyse von mehr als 150.000 USBürgern, die im vergleichbaren Zeitraum über eine App (Achievement EVIDATION, Evidation Health, Inc. San Mateo, CA, USA), die mehr als 20 Fitnesstracker zusammenführt, wissenschaftlich auswertet und ein gesundheitsförderndes Verhalten belohnt. Über alle 50 Staaten und Columbia hat sich eine Abnahme der täglichen Aktivität von mehr als $30 \%$ gezeigt, in 9 Bundesstaaten sogar um mehr als $50 \%$. EVIDATION ist im Dezember 2020 eine Partnerschaft mit dem American College of Cardiology zur Förderung der kardiovaskulären Gesundheit eingegangen (https://evidation.com/news/covid-19pulse-first-data-evidation [6]).

\section{Gemeinsamer Bundesaus- schuss - Telemonitoring bei Herzinsuffizienz}

Erstmalig hat der Gemeinsame Bundesausschuss (G-BA) nach langem Bewertungsprozess einen Zusatznutzen für die telemedizinische Begleitung von Herzinsuffizienz bestätigt. In seinem Beschluss vom 17.12.2020 hat der G-BA festgelegt, dass die lückenlose telemedizinische $\mathrm{Be}$ treuung von Patienten mit fortgeschrittener Herzschwäche zum ambulanten Leistungsangebot der gesetzlichen Krankenkassen gehören wird und somit nur durch zugelassene Kassenärzte und Ermächtigte ausgeübt werden darf.

In enger Kooperation soll die Analyse der in das telemedizinische Zentrum (TMZ) übertragenen Daten aus Implantaten, aber auch von externen Geräten durch das internistisch/kardiologische Personal erfolgen, die Therapieentscheidung und -umsetzung durch die niedergelassenen Ärzte. Das TMZ ist für die Datenanalyse, die technische Ausstattung der Patienten und für die Versorgung zu Zeiten, bei denen Praxen geschlossen haben, verantwortlich.

Als Indikation für ein Telemonitoring ist eine Herzinsuffizienz im Stadium
NYHA (New York Heart Association) II oder NYHA III mit einer linksventrikulären Auswurffraktion (LVEF) von < $40 \%$ anzusehen.

Die vom G-BA herangezogenen Studien mit positiven Endpunkten basierten zum einen auf Daten implantierter Aggregate $[7,8]$ oder externer Geräte $[9,10]$. Allen gemeinsam ist, dass klar strukturierte Abläufe mit hohem Personalaufwand und eindeutig hinterlegte Analyse- und Behandlungsalgorithmen einzuhalten waren. Zahlreiche andere Studien sind an uneindeutigen Strukturen gescheitert und haben keinen Nutzen der Telemedizin für herzinsuffiziente Patienten belegen können.

Die kurze Zusammenfassung der Eckpunkte des G-BA-Beschlusses dürfte klarmachen, welches Spannungsfeld sich auch für rhythmologische Patienten aufbauen wird, da zum einen höchste internistisch-kardiologische Kompetenz im TMZ gefordert wird, eine Genehmigung der Kassenärztlichen Vereinigung im entsprechenden Versorgungsgebiet vorliegen muss, die therapeutische Umsetzung in die Zuständigkeit des/der PBA (primär behandelnden/er Arztes/in) fällt und die finanzielle Vergütung an die Ermächtigung gekoppelt ist. Die Rolle der Kliniken, der überregionalen Herzinsuffizienzzentren und der Herzinsuffizienzschwerpunktpraxen ist nicht definiert. Von diesen ist bisher über Jahre die telemedizinische Versorgung insbesondere bei implantatgebundener Datenübermittlung nahezu unentgeltlich sichergestellt und diese Technologie auch weiterentwickelt worden.

\section{Studien in der Device-Therapie}

Die besondere pandemiebedingte Situation im Jahr 2020 und auch im Jahr 2021 hat - wie beschrieben - auch Auswirkung auf die Publikation neuer, nicht COVIDassoziierter kardiologischer Daten sowie auf die Durchführung bereits laufender Studien, nicht nur im Bereich rhythmologischer Schwerpunkte.

Es sind keine neuen Guidelines oder Empfehlungen zur Device-Therapie erschienen. In den aktuellen 2020 ESC (European Society of Cardiology) Guidelines zur Diagnose und Therapie von Vorhof- flimmern [11] finden Herzschrittmacher außer zur beiläufigen Diagnose von atrialen Hochfrequenzepisoden keine weitere Erwähnung. Multizentrische internationale Landmark-Trials aus dem Bereich der Device-Therapie, die unser Denken und Handeln beeinflussen könnten, sind ebenso nicht erschienen.

Die nächste klinisch bedeutsame Studie könnte RESET-CRT sein (Reevaluation of Optimal Re-synchronisation Therapy in Patients With Chronic Heart Failure) (ClinicalTrials.gov Identifier: NCT03494933). Diese beabsichtigt zu klären, ob eine CRT-P(Cardiac Resynchronization Therapy-Pacemaker)Therapie bei Patienten mit optimal medikamentös behandelter Herzinsuffizienz einer CRT-D(Cardiac Resynchronization Therapy-Defibrillator)-Therapie in Bezug auf "all-cause-mortality“ nicht unterlegen ist. Start war im August 2018, 1800 Patienten sollen auf CRT-P oder CRT-D randomisiert werden, 35 Monate sind für den Einschluss geplant, die finale Analyse ca. 10 Monate später, sodass im April 2022 die Daten zur Auswertung vorliegen könnten, COVID bedingt doch eher später.

\section{Sondenlose Herzschrittmacher}

Seit nunmehr bald 65 Jahren werden Herzschrittmachersysteme implantiert, die immer mit einer oder mehreren Sonden im Herzen oder auf dem Herzen stimulieren konnten und somit Leben retten. Die häufigsten Probleme gehen von den Elektroden oder der Schrittmachertasche aus und erfordern zum Teil komplexe und risikoreiche Extraktionsmanöver.

Hieraus ist der Wunsch entstanden, kabellose Schrittmacher zu entwickeln, die über einen Katheter implantiert werden, komplett im Herzen verbleiben und möglichst miniaturisiert sind.

Im Juli 2015 erfolgte die CE-Zulassung und damit die offizielle Marktzulassung des ersten über die Femoralvene in Kathetertechnik („transcatheter pacemaker systems“ [TPS]) einzuführenden „Kapsel“-Schrittmachers. Dieser wird in der rechten Herzkammer platziert und mit Widerhaken im Trabekelwerk verankert. Auch wenn inzwischen weltweit mehr als 


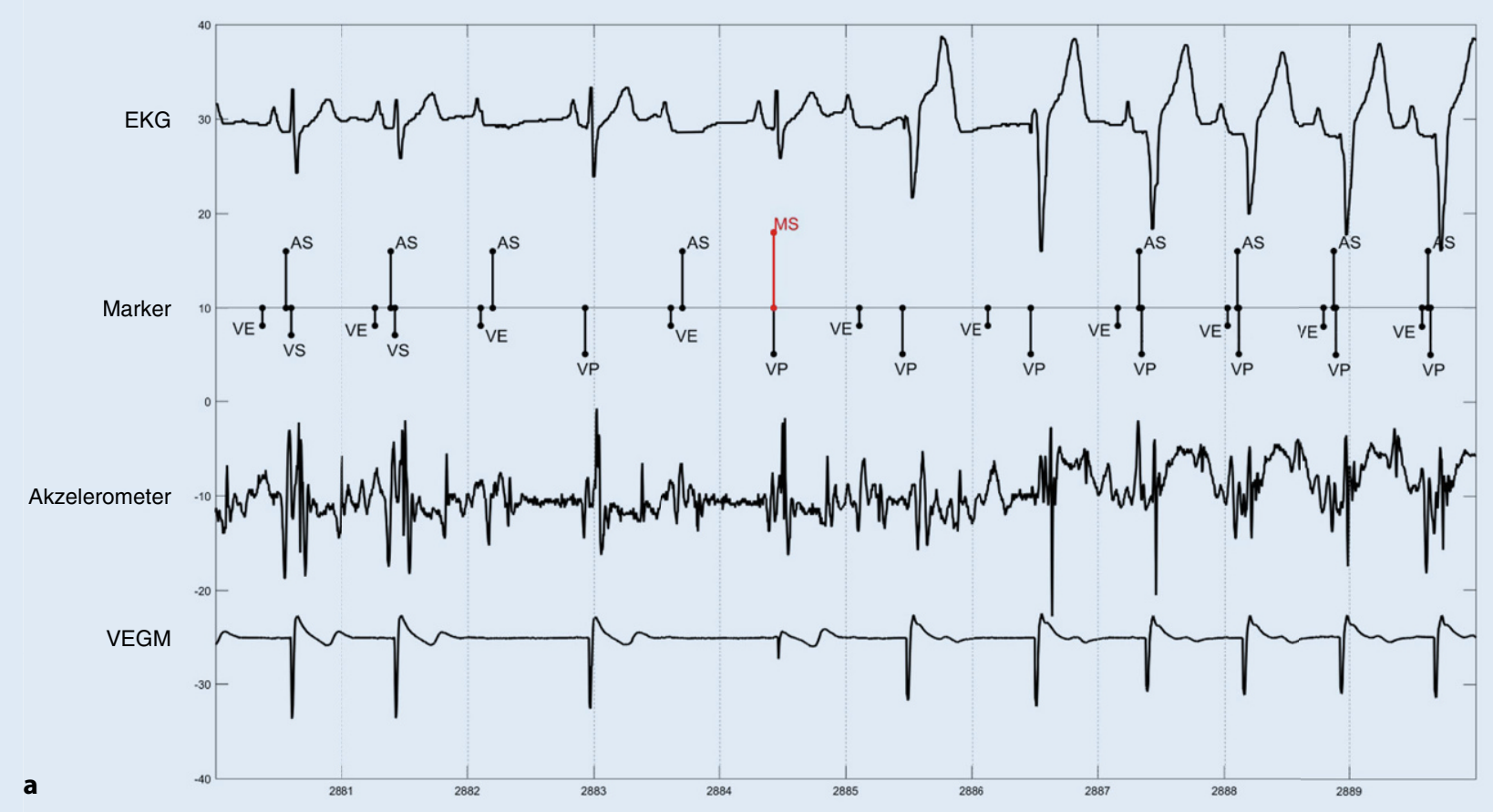

a

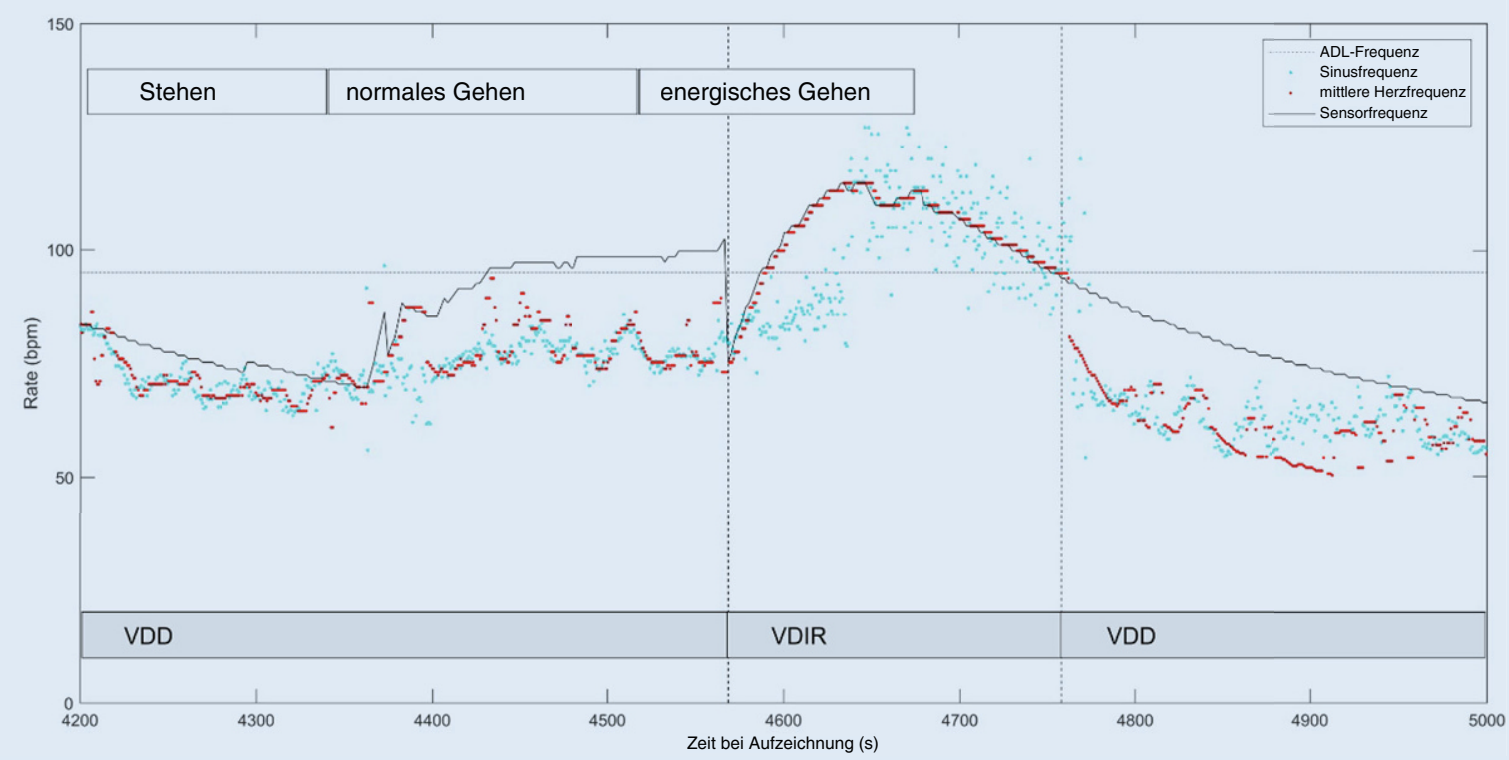

b

Abb. $1 \Delta$ a AV(atrioventrikulär)-Überleitungs-Moduswechsel bei einem Patienten mit intermittierendem AV-Block III. Grades. Vor dem Moduswechsel bei 2884 s weist der Patient eine AV-Überleitung im VVI(ventrikuläre Einkammerstimulation)40-Modus auf. Dann tritt bei dem Patienten ein AV-Block II. Grades auf, und der Gerätemodus wechselt zu VDD (Vorhofgetriggerte Ventrikelstimulation). Zwei Zyklen nach dem Moduswechsel beginnt das Gerät das Tracking des Vorhofs im VDDModus. b Aktivitätsmoduswechsel bei einem Patienten mit vollständigem AV-Block und normalem Sinusrhythmus. Während der Steh-und/oder Gehmanöver wird eine gute AV-Synchronizität durch die Ähnlichkeit der mittleren Herzfrequenz (HR) und der Sinusfrequenzangezeigt. Der Moduswechsel zu VVIR tritt ein, wenn die Sensorfrequenz sich von der Stimulationsfrequenz unterscheidet. Die Sensorfrequenz sinkt bis zur mittleren HR und steigt dann schrittweise, um große Frequenzänderungen zu minimieren. Nachdem die Aktivität aufgehört hat, geht der Modus wieder in VDDzurück, um so ein AV-synchrone Stimulation zu fördern. $A D L$ Aktivitäten des täglichen Lebens, $A S$ atriale Signalerkennung, bpm Schläge pro Minute, EKG Elektrokardiogramm, MS Moduswechsel, VE ventrikuläres Ende, VEGM ventrikuläres Elektrogramm, VP ventrikuläre Stimulation, VS ventrikuläre Signalerkennung 


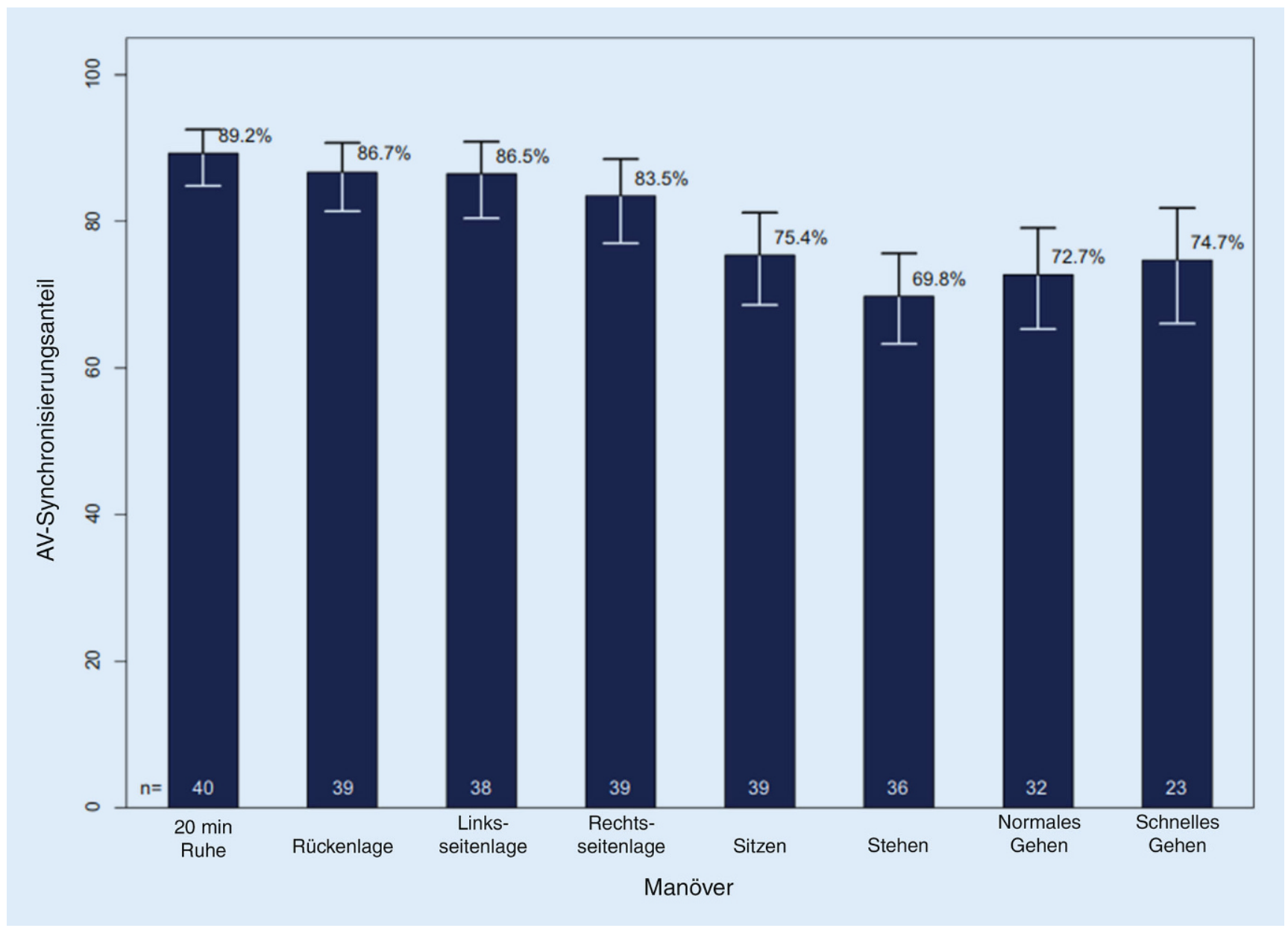

Abb. 2 ^ AV-Synchronisierungsprozentsatz nach Manöver [14]

50.000 solcher Systeme implantiert worden sind, ist sein Einsatz begrenzt, da er nur über eine VVI(R)-Stimulationsfunktion verfügt und bei sehr selten auftretenden AV-Blockierungen überhaupt eingesetzt werden kann.

Die Notwendigkeit einer AV-sequenziellen Stimulation mündete in der CEZulassung und Marktzulassung des Micra AV (Medtronic Parkway, MN, USA) $[12,13]$ im Juli 2020, der ebenso nur im rechten Ventrikel (RV) implantiert wird. Bei gleicher Größe wird über den Akzelerometer die mechanische Kontraktion des rechten Vorhofs wahrgenommen und eine VDD(Vorhofgetriggerte Ventrikelstimulation)-Stimulation veranlasst. Eine atriale Stimulation ist nicht möglich. Der Algorithmus nutzt das mechanische Signal der passiven ventrikulären Füllung (A3) und der aktiven atrialen Kontraktion (A4) sowie 2 Mode-switch-Algorith- men, die zu einer VVI-40- und VVIRStimulation führen.

In MARVEL 2 konnte gezeigt werden, dass bei intermittierendem AVBlock III zuerst eine VVI-Back-upStimulation stattfindet (-Abb. 1), bevor nach einigen wahrgenommenen Vorhofaktionen eine AV-Synchronizität erreicht wird (•Abb. 2; [14]). Die erreichbare AV-Synchronizität war lageund belastungsabhängig und erreichte bei längerer Ruhe $89 \%$, sank aber beim Stehen und Laufen auf ca. 70\% [14]. Echokardiographische Parameter, wie z. B. ein E/A-Verhältnis $<0,94$ und eine geringe Variabilität der Sinusrate in Ruhe, korrelieren mit einer hohen AVsequenziellen Stimulationsrate [15].

Hieraus ergeben sich die Fragen: Wie viel AV-synchrone Stimulation ist notwendig, wie werden die asynchronen Phasen klinisch wahrgenommen, was passiert bei stärkerer Belastung, wie ist die Wahrnehmung bei deutlich vergrößertem, mechanisch eher inaktivem rechtem Vorhof, und welche Bedeutung hat eine Trikuspidalinsuffizienz? Folglich wird das Patientenklientel für die Zukunft zu definieren sein.

\section{Primärprävention des plötzlichen Herztodes - mehr als linksventrikuläre Auswurffraktion}

Der plötzliche Herztod (SCD) stellt unverändert eine bedeutsame Bedrohung dar, auch wenn sich aktuell in den nationalen und internationalen Empfehlungen keine Änderung ergeben hat. Gerade die moderne medikamentöse Herzinsuffizienztherapie mit vielen neuen, sich vermutlich zeitnah etablierenden Präparaten wie SGLT2-Inhibitoren und der frühzeitigere Einsatz der ARNIs (Angiotensin-Rezeptor-Neprilysin-In- 


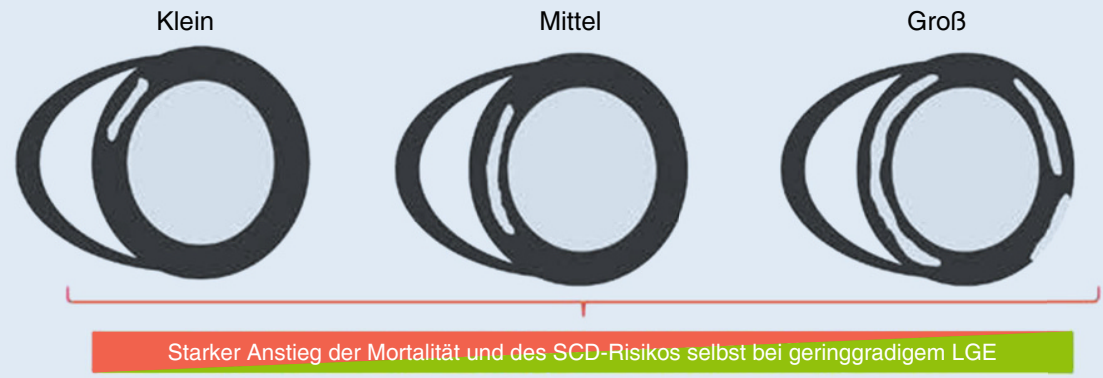

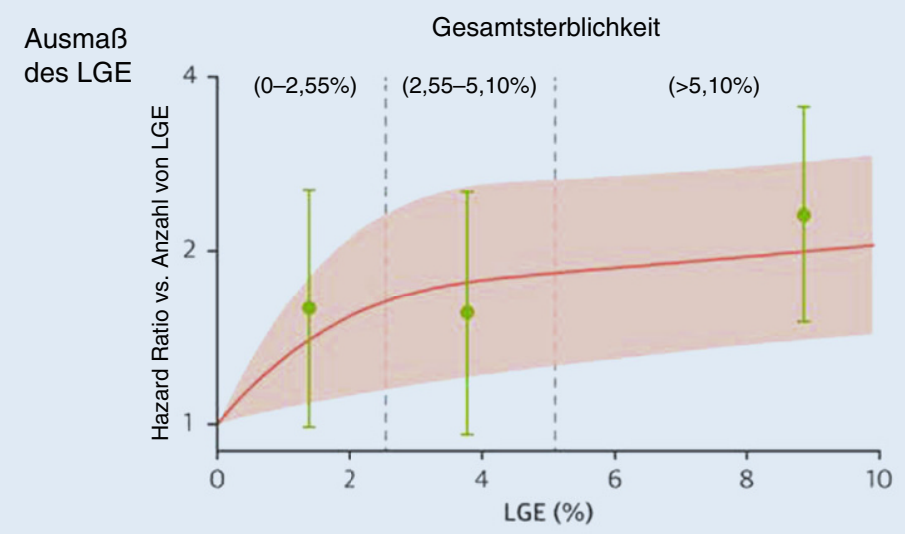

Gesamtsterblichkeit: adjustiert in Bezug auf LVEF, Alter und Geschlecht

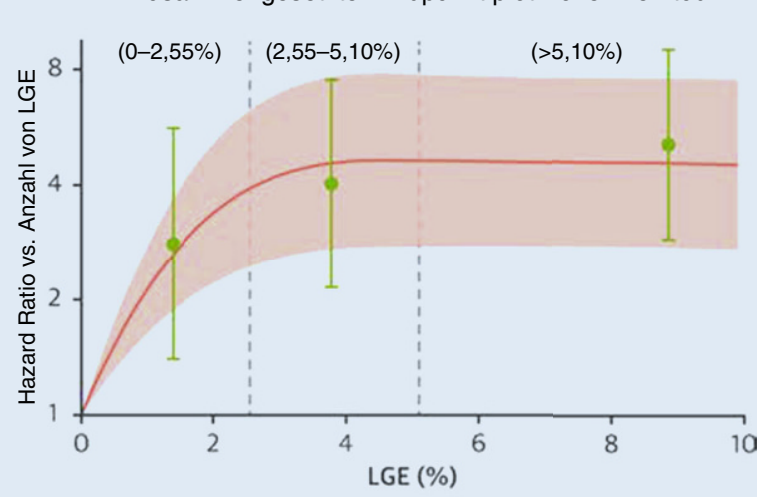

SCD/ASCD: adjustiert in Bezug auf LVEF, Alter und Geschlecht Kategoriale HR

LGE-

Lokalisierung
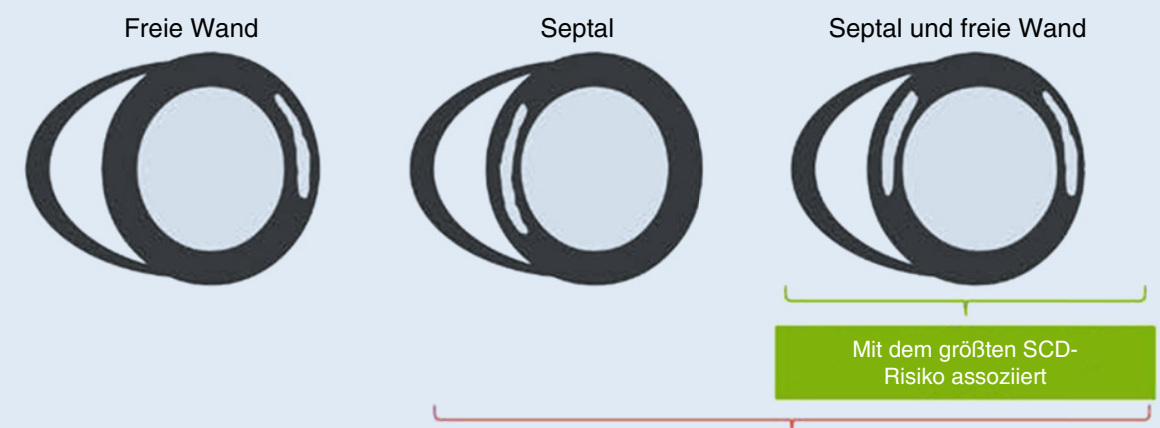

Septales LGE mit erhöhter Sterblichkeit assoziiert

LGE-

Muster

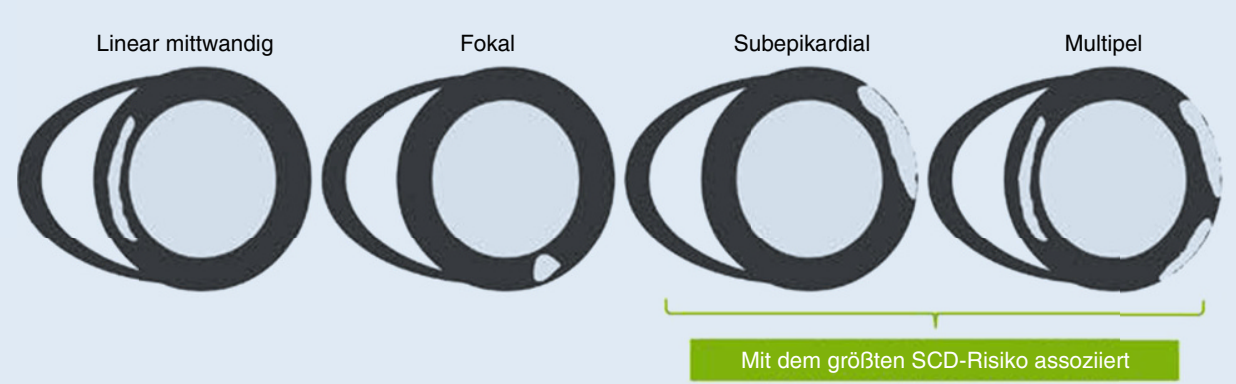

Relevanz für Sterblichkeitsrisiko Relevanz für SCD-Risiko

Abb. 3 ॥ "Late gadolinium enhancement" (LGE) und Outcome bei Patienten mit dilatativer Kardiomyopathie. LVEF linksventrikuläre Ejektionsfraktion, SCD plötzlicher Herztod („,sudden cardiac death“),ASCD atherosklerotische kardiovaskuläre Erkrankungen („atherosclerotic cardiovascular disease“). (Mod. nach [18]) 
hibitoren), die Erkenntnis über eine deutlich längere Herzinsuffizienzvorbehandlung bis $\mathrm{zu} 6$ Monaten und die Überbrückung einer initial rhythmologisch besonders vulnerablen Phase durch einen „Wearable-Defibrillator“ stellen die Notwendigkeit einer permanenten ICDImplantation zunehmend infrage bzw. erfordern andere Selektionskriterien als die alleinige linksventrikuläre Ejektionsfraktion [16]. Eine zunehmende Bedeutung gewinnt dabei die Bildgebung, insbesondere die MRT, bei der Erkennung von intramuralem Narbengewebe und dessen Verteilungsmuster. In einer prospektiven Untersuchung von mehr als 1000 Patienten mit nichtischämischer Kardiomyopathie (NICM) mit einem medianen Follow-up von mehr als 5 Jahren waren die LVEF $<35 \%$ und Narbennachweis signifikant mit der Gesamt- und kardialen Sterblichkeit assoziiert, aber nur die nachgewiesene Narbe war mit dem SCD assoziiert, die LVEF jedoch nicht [17]. Die Narbensuche (in der MRT) sollte in die Patientenselektion aufgenommen werden.

In der bisher größten Untersuchung an über 800 Patienten, die ebenso an einer NICM erkrankt waren, konnte gezeigt werden, dass sowohl der Nachweis von Narben als „late gadolinium enhancement" (LGE) als auch das intramurale Verteilungsmuster relevant sind für die Gesamtsterblichkeit und den plötzlichen Herztod. Eine septale und laterale Wandbeteiligung sowie multiple als auch subepikardiale Spots gehen mit dem höchsten Risiko einher, am plötzlichen Herztod zu versterben (- Abb. 3; [18]).

Auch bei ischämischer Kardiomyopathie (ICM) von fast 1000 Patienten, bei denen die "grey zone“ der myokardialen Fibrose in der MRT visuell festgestellt wurde und in ihrer Masse quantifiziert wurde, war der Narbennachweis mit mehr als $5 \mathrm{~g}$ mit einer HR (Hazard Ratio) von 10,8 zum SCD korreliert, eine LVEF $<35 \%$ korrelierte deutlich schwächer. Somit wird auch in dieser aktuellen Studie der Wert der Narbendetektion bei ICM hervorgehoben und scheint der LVEF deutlich überlegen [19].

Zusammenfassend sollte von Elektrophysiologen und Implanteuren vermehrt eine über die alleinige LVEF-
Bestimmung hinausgehende Bildgebung insbesondere zur Narbendetektion in der MRT angeregt werden, um diese Informationen dann bei der Entscheidungsfindung zur Notwendigkeit einer ICD-Implantation zu berücksichtigen.

\section{Schlussfolgerung}

Die große Herausforderung wird nicht nur in der Kardiologie darin bestehen, durch die Pandemie bedingte Verunsicherungen aufzulösen und Vertrauen wiederzugewinnen. Rhythmologische Eingriffe, die nicht der Beseitigung einer akuten Notfallsituation dienen und einen für den Patienten eher prognostischen, nicht direkt greifbaren Wert haben, werden bezüglich ihrer Notwendigkeit und Dringlichkeit hinterfragt immer in der Sorge, sich in einer Ambulanz, einer Praxis oder der Klinik einer COVID-Infektion auszusetzen und sich und Angehörige zu gefährden. Hierdurch bedingt, sind in nunmehr einem ganzen Jahr Maßnahmen unterblieben, die es gilt, wohldosiert, gestaffelt und unter Beibehaltung höchster Sicherheitsmaßnahmen nachzuholen und eine entstandene Unterversorgung auszugleichen.

Sicher sind Probleme, wie z. B. Infektionen, Perforationen an Schrittmacherund Defibrillatortaschen, durch mangelnde Präsenzbesuche verspätet oder nicht entdeckt worden. Hierauf sollte unsere vermehrte Wachsamkeit in Zukunft gerichtet sein.

Die Pandemie hätte der Telemedizin in der Device-Therapie, dem RemoteMonitoring und der Televisite einen substanziellen Schub verschaffen können, der aber gefühlt nicht eingesetzt hat. Die Strukturen sind noch nicht übergreifend vorhanden, klare sektorenübergreifende Technologien und Behandlungsrichtlinien sind nicht ausreichend etabliert. Ebenso sollte die telemedizinische Vergütung alle Beteiligten in der rhythmologischen Versorgung gleichermaßen berücksichtigen und somit auch motivieren.

Auch unter erschwerten Bedingungen sollten unsere Anstrengungen nicht nachlassen, die aktuellen Milestone-Studien, wie z. B. RESET-CRT, voranzubrin- gen, um unseren Patienten bei zu erwartenden geringeren Ressourcen evidenzbasiert die notwendige, aber nicht überschießende Therapie zukommen zu lassen. Gerade unter der sich zurzeit vehement im Fluss befindlichen medikamentösen und interventionellen Herzinsuffizienztherapie erscheinen viele unserer im Bewusstsein tief verankerten, richtungsweisenden historischen Studien nicht mehr kritiklos anwendbar.

\section{Fazit für die Praxis}

- Die COVID-Pandemie hat zu einem deutlichen Rückgang der körperlichen Aktivität und einer Abnahme bis zu $50 \%$ in der Versorgung mit Herzschrittmacher/ Defibrillatorimplantaten sowie Ablationen geführt. Diese minderversorgten Patienten sollten im Fokus unserer Aufmerksamkeit stehen.

- Patienten mit höhergradigen AV(atrioventrikulären)-Blockierungen kann jetzt die Therapieoption mit einem kabellosen miniaturisierten Schrittmacher angeboten werden, der nur im rechten Ventrikel über eine Katheterprozedur implantiert wird. Ob der zugrunde liegende herztonbasierte VAT(vorhofgetriggerte ventrikuläre Stimulation)-Algorithmus alltagstauglich ist und eine klinisch zufriedenstellende AVSynchronizität ermöglicht, wird die Zukunft zeigen.

- Die Notwendigkeit einer primärprophylaktischen ICD(implantierbarer Kardioverter-Defibrillator)-Implantation sollte gerade im Bereich der nichtischämischen Kardiomyopathie eine diagnostische Erweiterung über die LVEF(linksventrikuläre Auswurffraktion)-basierte Einschätzung hinaus durch den intramuralen Narbennachweis und das Verteilungsmuster erfahren. 


\begin{tabular}{|l}
\hline Prof. Dr. Christian Butter \\
Immanuel Klinikum Bernau \\
Herzzentrum Brandenburg, \\
Universitätsklinikum der \\
Medizinischen Hochschule \\
Brandenburg \\
Ladeburger Str. 17, \\
16321 Bernau bei Berlin, \\
Deutschland \\
christian.butter@ \\
immanuelalbertinen.de
\end{tabular}

\section{Einhaltung ethischer Richtlinien}

Interessenkonflikt. C. Butter und J. Sperzel geben an, dass kein Interessenkonflikt besteht.

Für diesen Beitrag wurden von den Autoren keine Studien an Menschen oder Tieren durchgeführt. Für die aufgeführten Studien gelten die jeweils dort angegebenen ethischen Richtlinien.

\section{Literatur}

1. Harper L, Kalfa N, Beckers GMA et al (2020) The impact of COVID-19 on research. J Pediatr Urol 16:715-716

2. Lakkireddy DR, Chung MK, Gopinathannair R et al (2020) Guidance for cardiac electrophysiology during the COVID-19 pandemic from the Heart Rhythm Society COVID-19 Task Force; Electrophysiology Section of the American College of Cardiology; and the Electrocardiography and Arrhythmias Committee of the Council on Clinical Cardiology, American Heart Association. Heart Rhythm 17:e233-e241

3. Slotwiner D, Varma N, Akar JG et al (2015) HRS Expert Consensus Statement on remote interrogation and monitoring for cardiovascular implantable electronic devices. Heart Rhythm 12:e69-e100

4. Boriani G, Palmisano P, Guerra F et al (2020) Impact of COVID-19 pandemic on the clinical activities related to arrhythmias and electrophysiology in Italy: results of a survey promoted by AIAC (Italian Association of Arrhythmology and Cardiac Pacing). Intern Emerg Med 15:1445-1456

5. Sassone B, Mandini S, Grazzi G et al (2020) Impact of COVID-19 Pandemic on Physical Activity in Patients with Implantable Cardioverter-Defibrillator. JCardiopulm Rehabil Prev 40:285-286

6. (2020) https://evidation.com/news/covid-19pulse-first-data-evidation/. Zugegriffen: 18. Febr. 2021

7. Hindricks G, Taborsky M, Glikson M et al (2014) Implant-based multiparameter telemonitoring of patients with heart failure (IN-TIME): a randomised controlled trial. Lancet 384:583-590

8. Sardu C, Santamaria M, Rizzo M et al (2016) Telemonitoring in heart failure patients treated by cardiac resynchronisation therapy with defibrillator (CRT-D): the TELECART Study. Int J Clin Pract 70:569-576

9. Koehler F, Winkler S, Schieber M et al (2011) Telemedical Interventional monitoring in heart failure investigators. Circulation 123:1873-1880
10. Koehler F, Koehler K, Deckwart O et al (2018) Efficacy of telemedical interventional management in patients with heart failure (TIM-HF2): a randomised, controlled, parallel-group, unmasked trial. Lancet 392:1047-1057

11. Hindricks G, Potpara T, Dagres N et al (2021) 2020 ESC Guidelines for the diagnosis and management of atrial fibrillation developed in collaboration with the European Association for Cardio-Thoracic Surgery (EACTS). Eur Heart J 42:373-498

12. Chinitz L, Ritter P, Khelae SK et al (2014) Accelerometer-based atrioventricular synchronous pacing with a ventricular leadless pacemaker: results from the Micra atrioventricular feasibility studies. Heart Rhythm 15:1363-1371

13. Garweg C, SplettV, Sheldon TJet al (2019) Behavior of leadless AV synchronous pacing during atrial arrhythmias and stability of the atrial signals over time-Results of the MARVEL Evolve subanalysis. Pacing Clin Electrophysiol 42:381-387

14. Steinwender C, Khelae SK, Garweg C et al (2020) Atrioventricular synchronous pacing using a leadless ventricular pacemaker: results from the MARVEL 2 study. JACC Clin Electrophysiol 6:94-106

15. Garweg C, Khelae SK, Steinwender C et al (2020) Predictors of atrial mechanical sensing and atrioventricular synchrony with a leadless ventricular pacemaker: results from the MARVEL 2 study. Heart Rhythm 17:2037-2045

16. Wong JA, Roberts JD, Healey JS (2021) The optimal timing of primary prevention implantable cardioverter-defibrillator referral in the rapidly changing medical landscape. Can J Cardiol S0828 282X(21)00069-6. https://doi.org/10.1016/j.cjca. 2021.01.024

17. Klem I, Klein M, Khan Metal (2021) The relationship of LVEF and myocardial scar to long-term mortality risk and mode of death in patients with nonIschemic cardiomyopathy. Circulation. https://doi. org/10.1161/CIRCULATIONAHA.120.048477

18. Halliday BP, Baksi J, Gulati A et al (2019) Outcome in dilated cardiomyopathy related to the extent, location, and pattern of late gadolinium enhancement. JACC CardiovasC Imaging 12:1645-1655

19. Zegard A, Okafor O, de Bono J et al (2021) Myocardial fibrosis as a predictor of sudden death in patients with coronary artery disease. J Am Coll Cardiol 77:29-41

\section{Arrhythmien in der Schwangerschaft}

Die Behandlung Schwangerer stellt in der täglichen internistischen und kardiologischen Praxis nicht unbedingt ein zentrales Thema dar. Daher kann beim unvorbereiteten Vorstellen einer Schwangeren mit Herzrhythmusstörungen eine gewisse Unsicherheit entstehen. Und dies kann häufiger werden: Relevante Arrhythmien in der Schwangerschaft sind zwar insgesamt selten, treten aber zunehmend öfter auf, da Schwangere in Deutschland älter werden und trotz bekannter Herzerkrankung konsequenter einem Kinderwunsch nachgegangen wird.

Heft 2/2021 von Herzschrittmachertherapie und Elektrophysiologie stellt Beiträge vor, die bei der Behandlung und Beratung von Patientinnen mit arrhythmogenen Erkrankungen, die schwanger sind oder einen Kinderwunsch äußern, helfen sollen. Zentral ist dabei neben zahlreichen Tipps und Tricks nicht zuletzt die enge Kooperation mit Gynäkologie und Anästhesie.

- Up-to-date Informationen zu einem oft vernachlässigten Thema

- 12 Beiträge von Expert*innen zur fundierten Behandlung und Beratung von Schwangeren mit arrhythmogenen Erkrankungen, u.a.: Antiarrhythmika - Sicherheit und Wirksamkeit in der Schwangerschaft; Fetales EKG und Arrhythmien; Schwangerschaft und Vorhofflimmern; Bradykardie in der Schwangerschaft; Sowie: Invasive Mapping- und Ablationsprozedur; angeborene Herzfehler; Long-QT-Syndrom Arrythmogene Kardiomyopathie; katecholaminerge polymorphe ventrikuläre Tachykardie EKG und Arrhythmien bei Peripartumkardiomyopathie; ICD

Herzschrittmachertherapie + Elektrophysiologie 2/2021 | springermedizin.de 